\title{
Hemoglobin level predicts outcome for vulvar cancer patients independent of GLUT-1 and CA-IX expression in tumor tissue
}

\author{
Hedwig P. van de Nieuwenhof • Joanne A. de Hullu • \\ Johannes H. A. M. Kaanders • Johan Bulten • \\ Leon F. A. G. Massuger • Léon C. L. T. van Kempen
}

Received: 28 July 2010 /Revised: 1 September 2010 / Accepted: 12 September 2010 /Published online: 2 October 2010

(C) The Author(s) 2010. This article is published with open access at Springerlink.com

\begin{abstract}
Intratumoral hypoxia has been associated with poor prognosis in several solid tumors. The aim of this study was to determine whether the hypoxia-associated markers glucose transporter (GLUT)-1 and carbonic anhydrase (CA)-IX expression and preoperative hemoglobin $(\mathrm{Hb})$ levels correlate with presence of inguinofemoral or distant metastases, and disease-free survival (DSS) in vulvar squamous cell carcinoma (SCC) patients. Vulvar SCC $(n=103)$ were reviewed for histopathological characteristics by an expert gynecopathologist and stained for GLUT-1 and CA-IX. Clinical data and preoperative $\mathrm{Hb}$ levels were obtained from medical records. No significant correlations were observed between GLUT-1 or CA-IX expression patterns and preoperative $\mathrm{Hb}$ levels, presence of inguinofemoral or distant metastases and DSS. However, anemic patients $(\mathrm{Hb}<11.2 \mathrm{~g} / \mathrm{dL})$ had significantly more inguinofemoral metastases and lower $\mathrm{Hb}$ level was an independent prognostic factor for a worse DSS $(p<0.001)$. The number of comorbidic conditions was inversely correlated with preoperative $\mathrm{Hb}$ level. Preoperative $\mathrm{Hb}$
\end{abstract}

H. P. van de Nieuwenhof $(\bowtie) \cdot$ J. A. de Hullu •

L. F. A. G. Massuger

Departments of Obstetrics and Gynecology,

Radboud University Nijmegen Medical Centre,

P.O. Box 9101, 6500 HBNijmegen, The Netherlands

e-mail: H.Nieuwenhof@obgyn.umcn.nl

J. H. A. M. Kaanders

Department of Radiation Oncology,

Radboud University Nijmegen Medical Centre,

Nijmegen, The Netherlands

J. Bulten • L. C. L. T. van Kempen

Department of Pathology,

Radboud University Nijmegen Medical Centre,

Nijmegen, The Netherlands levels are associated with poor DSS for vulvar SCC patients, whereas tumor hypoxia reflected by GLUT-1 and CA-IX expression does not have a predictive value. Because preoperative $\mathrm{Hb}$ levels inversely correlated with the number of comorbidic conditions and not with GLUT-1 or CA-IX expression, it is most likely that preoperative $\mathrm{Hb}$ levels represent overall physical condition.

Keywords Vulvar squamous cell carcinoma Prognosis . Hypoxia $\cdot$ Anemia $\cdot$ Comorbidity

\section{Introduction}

Vulvar squamous cell carcinoma (SCC) accounts for 3-5\% of all gynecological malignancies. Positive inguinofemoral lymph nodes have been found to be the most important prognostic factor for vulvar SCC [1-3]. Currently, the standard treatment for patients with early stage vulvar SCC is wide local excision with uni- or bilateral inguinofemoral lymphadenectomy via separate incisions that may lead to impressive morbidity such as lymphedema. There are no non-invasive diagnostic tests that are able to exclude inguinofemoral lymph node metastasis with a high enough certainty to safely omit a complete inguinofemoral lymphadenectomy [4]. Fortunately, the sentinel lymph node procedure is an upcoming promising staging technique [5].

Limited research has been performed on immunohistochemical markers to predict the presence of positive inguinofemoral lymph nodes and the prognosis of the individual patient with vulvar SCC. Fons et al. have analyzed 16 immunohistochemical markers with potentially prognostic value for patients with vulvar SCC. Lack of caspase three expression, a key effector of cellular death, 
was associated with poor disease-specific survival (DSS) in a multivariate analysis [6]. In addition, increased epidermal growth factor receptor (EGFR) expression and increased angiogenic response have been found to be associated with decreased survival [7-11].

Hypoxia occurs in anemia [12], cancer, prolonged exercise, and long-term ischemia with durations of several hours or more [13]. Hypoxia may induce cell death, although tumor cells may adapt to the hypoxic microenvironment by inducing a hypoxia-inducible factor (HIF)-1 mediated angiogenic response, and/or by altering their metabolic activity [14]. The overall cellular consequences of hypoxia are a decreased proliferation rate, increased radio- and multidrug resistance, and increased invasive and metastatic potential [15]. These adaptive responses promote tumor progression and treatment resistance to both radioand chemotherapy. Several studies have shown that tumor hypoxia is significantly related to poor patient prognosis in various malignancies such as head and neck cancer, and cervical cancer $[16,17]$.

Several immunohistochemical markers can assess hypoxia. HIF-1 is considered a master regulator of the cellular response to hypoxia. However, although HIF-1 indicates tumor aggressiveness, it is debatable whether it is also a reliable marker of tumor hypoxia as HIF1 induction is also dependent on other microenvironmental conditions [14]. Among others, HIF-1 regulates the expression of the surface transmembrane glucose transporters (GLUTs), especially GLUT-1 and carbonic anhydrases (CAs) which are both involved in energy metabolism [18-21]. Whereas GLUTs import glucose across the cell membrane in an energy independent manner to fuel anaerobic glycolysis [22], the subsequent intracellular acidification is antagonized by CAs [23]. This results in an acidic extracellular environment that may promote progression toward a more malignant phenotype [24]. Of all CAs, CA-IX has the greatest magnitude of induction under hypoxic conditions.

Oxygen delivery to tumors depends on tissue perfusion and the oxygen-carrying capacity of the blood. It has been suggested that a decrease in the oxygen-carrying capacity of the blood caused by anemia may be a major causative factor for the development of tissue hypoxia $[25,26]$. In a rat model, Kelleher et al. indeed demonstrated that tumorrelated anemia resulted in substantial worsening of tumor oxygenation [27]. Both high CA-IX, GLUT-1 and low preoperative hemoglobin $(\mathrm{Hb})$ levels were found to be associated with a poor DSS in different tumor types, e.g., cervical, ovarian, and head and neck carcinomas [28-36].

Studies that have analyzed the correlation between hypoxia and prognosis in vulvar SCC are limited. In a small-scale study (20 patients), only preoperative anemia showed a correlation with nodal metastasis [37]. Therefore, the aim of this study is to determine whether GLUT-1 and
CA-IX expression and preoperative hemoglobin levels correlate with presence of inguinofemoral or distant metastases and DSS in a large group of vulvar SCC patients.

\section{Materials and methods}

From January 1st 1988 until December 31st 2005, 167 consecutive patients with vulvar SCCs were treated with a curative intent in the Radboud University Nijmegen Medical Centre. Only patients whose treatment consisted of primary surgery were included. Tumors with a depth of invasion of $1 \mathrm{~mm}$ or more in the operation specimen were selected for this study. A total number of 103 paraffinembedded primary vulvar SCCs that met the abovementioned criterion with long-term follow-up were available for this study (last month of follow-up-August 2008). All slides were reviewed for histopathological characteristics by an expert gynecopathologist (JB). Clinical data were obtained from medical records and comorbidity was registered in accordance with the Comorbidity Index of Mary E. Charlson [38].

All patients were staged according to the surgicopathological staging system for vulvar carcinoma of the International Federation of Gynecology and Obstetrics (FIGO). When a recurrence was diagnosed, the localization of the recurrence was registered as local, skin bridge, groin, pelvic, or distant. For analytical purposes, skin bridge recurrences were considered to be groin recurrences and pelvic recurrences considered to be distant recurrences.

\section{Immunohistochemistry}

Formalin-fixed paraffin-embedded tissue sections $(4 \mu \mathrm{m})$ were mounted onto Superfrost slides (Menzel-Gläser, Braunschweig, Germany) and dried overnight at $37^{\circ} \mathrm{C}$. Tissues were dewaxed in xylene, rehydrated through graded alcohols, and rinsed three times in phosphate-buffered saline (PBS; pH 7.4) for $10 \mathrm{~min}$. Following an antigen retrieval step [sodium citrate $(0.01 \mathrm{M} ; \mathrm{pH} 6.0), 95^{\circ} \mathrm{C}$, $10 \mathrm{~min}]$, tissue sections were preincubated with $20 \%$ normal goat serum in PBS and subsequently incubated overnight at $4^{\circ} \mathrm{C}$ with a polyclonal rabbit-anti-GLUT-1 (Dako, Glostrup, Denmark; 1:200) or rabbit-anti-CA-IX $(0.1 \mu \mathrm{g} / \mathrm{mL}$, kindly provided by Dr. E. Oosterwijk, Dept. of Experimental Urology, Radboud University Nijmegen Medical Centre). Antibodies were diluted in PBS containing $1 \%$ bovine serum albumin (BSA). Slides were rinsed in PBS for $10 \mathrm{~min}$ and incubated with biotinylated anti-rabbit $\operatorname{IgG}$ (Vector, Burlingame, CA, USA) for $30 \mathrm{~min}$ at room temperature (RT). A biotin-avidin peroxidase (CA-IX) or alkaline phosphatase (GLUT-1) complex was generated 
according to standard procedures (Vector). Peroxidase was visualized with 3-amino-9-ethylcarbazole (AEC, ScyTek, Logan, UT, USA) and briefly counterstained with Mayer's hematoxylin. Alkaline phosphatase was visualized with Fast Blue (20 mL Tris-HCl pH 8.2, 4 mg Naphtol AS-MX, 4.8 mg Levamisole, and $20 \mathrm{mg}$ Fast Blue BB salt) and counterstained with Nuclear fast red (Vector). Slides were mounted with Imsol (Klinipath, Duiven, The Netherlands) and subsequently with Permount (Fisher Scientific, Fairlawn, NJ, USA). Negative controls (buffer only) were included in each analysis.

For GLUT-1/CD31 double staining, the sodium-citrate antigen retrieval step and preincubation with normal serum was followed by incubation with monoclonal mouse-anti CD31 (Dako, clone JC70A, 1:20) overnight at $4^{\circ} \mathrm{C}$. After washing the slides with PBS for $10 \mathrm{~min}$, tissue sections were incubated with biotinylated antimouse IgG (Vector), an avidin-peroxidase complex (Vector) generated and visualized with AEC. Subsequently, the GLUT-1 staining protocol (Fast Blue) was performed as described above.

\section{Evaluation of staining}

Slides were scored (HvdN, LvK) blinded to clinicopathological data and results of other stainings. Because GLUT-1 and CA-IX function as transmembrane proteins, only membranous staining was considered a positive result. The percentage of positive cells and localization of staining was determined and categorized in the following manner: negative, less than $5 \%$ of tumor cells stained, between $5 \%$ and $25 \%$, between $25 \%$ and $75 \%$, and over $75 \%$. To avoid small numbers, for GLUT-1 staining, the only tumor with less than 5\% cells stained was grouped together with 5$25 \%$ and for CA-IX staining, tumors with over $75 \%$ tumor cells stained (three tumors) were grouped together in a category $\geq 25 \%$ tumor cells stained.

\section{Hemoglobin levels}

Preoperative hemoglobin levels were recorded from the medical charts. All hemoglobin levels were taken within 1 week prior to surgery.

\section{Statistical analysis}

Data were entered in a computerized database and analyzed using SPSS software (version 16.0.1 for Windows, SPSS). Patient and tumor characteristics were compared using $\chi^{2}$ and two-tailed Student's $t$ tests. Survival analysis was performed by the Kaplan-Meier product limit method, and survival rates were compared using log-rank statistics. Cox regression analysis was used for confounder analysis. All time-dependent outcomes were calculated from time of pathological diagnosis. Patient data were censored when a disease event had not occurred at the last follow-up. For the endpoint "presence of inguinofemoral or distant metastases", an event was defined as occurrence of inguinofemoral metastases or metastasis in a distant organ. For DSS, only deaths due to vulvar SCC were analyzed as events. Patients who are still alive or died due to other causes than vulvar SCC were censored at the date of last follow-up. Values of $p<0.05$ were considered significant.

\section{Results}

Primary treatment and staging

A total number of 30 patients underwent a wide local excision, 72 patients had a radical vulvectomy and for one patient an exenterative procedure was performed. Postoperative radiotherapy was given to 31 patients (30\%); no patients were treated with adjuvant chemotherapy.

Median age of the patients was 72 years (range 3192 years). Nine $(8.7 \%)$ patients had a FIGO stage I tumor, $43(41.7 \%)$ patients a stage II, $32(31.1 \%)$ patients a stage III, and 19 patients $(18.5 \%)$ a stage IV tumor.

\section{Expression of GLUT-1 and CA-IX}

To assess hypoxia in vulvar SCCs, we performed immunohistochemical analyses for the tissue hypoxia-associated markers GLUT-1 and CA-IX. The median area stained by GLUT-1 was $25 \%$ of the whole tumor. Often a diffuse staining of a large tumor area was observed, with a focally increased staining intensity (Fig. 1). Both cytoplasmic and membranous GLUT-1 staining was observed, but only membranous staining was considered positive staining. In several cases, GLUT-1 expression was observed directly adjacent to a blood vessel (Fig. 1e-f).

CA-IX staining was less pronounced than GLUT-1, with a median staining area of only $1 \%$ (Fig. 2). CA-IX staining was often observed at a distance from vessels.

\section{Hemoglobin levels}

With the aim to assess the role of anemia, preoperative hemoglobin levels were recorded from medical charts. Hemoglobin levels were available for 77 patients. Eight patients $(7.8 \%$ ) had a $\mathrm{Hb}$ value below $11.2 \mathrm{~g} / \mathrm{dL}$ and were considered to be anemic. Eight patients had a $\mathrm{Hb}$ level between 11.2 and $12.6 \mathrm{~g} / \mathrm{dL}$, and 15 patients had a Hb level between 12.6 and $13.4 \mathrm{~g} / \mathrm{dL}$. Twenty patients had a $\mathrm{Hb}$ level between 13.4 and $14.2 \mathrm{~g} / \mathrm{dL}$, and 26 patients had a $\mathrm{Hb}$ level of $14.2 \mathrm{~g} / \mathrm{dL}$ or higher. 


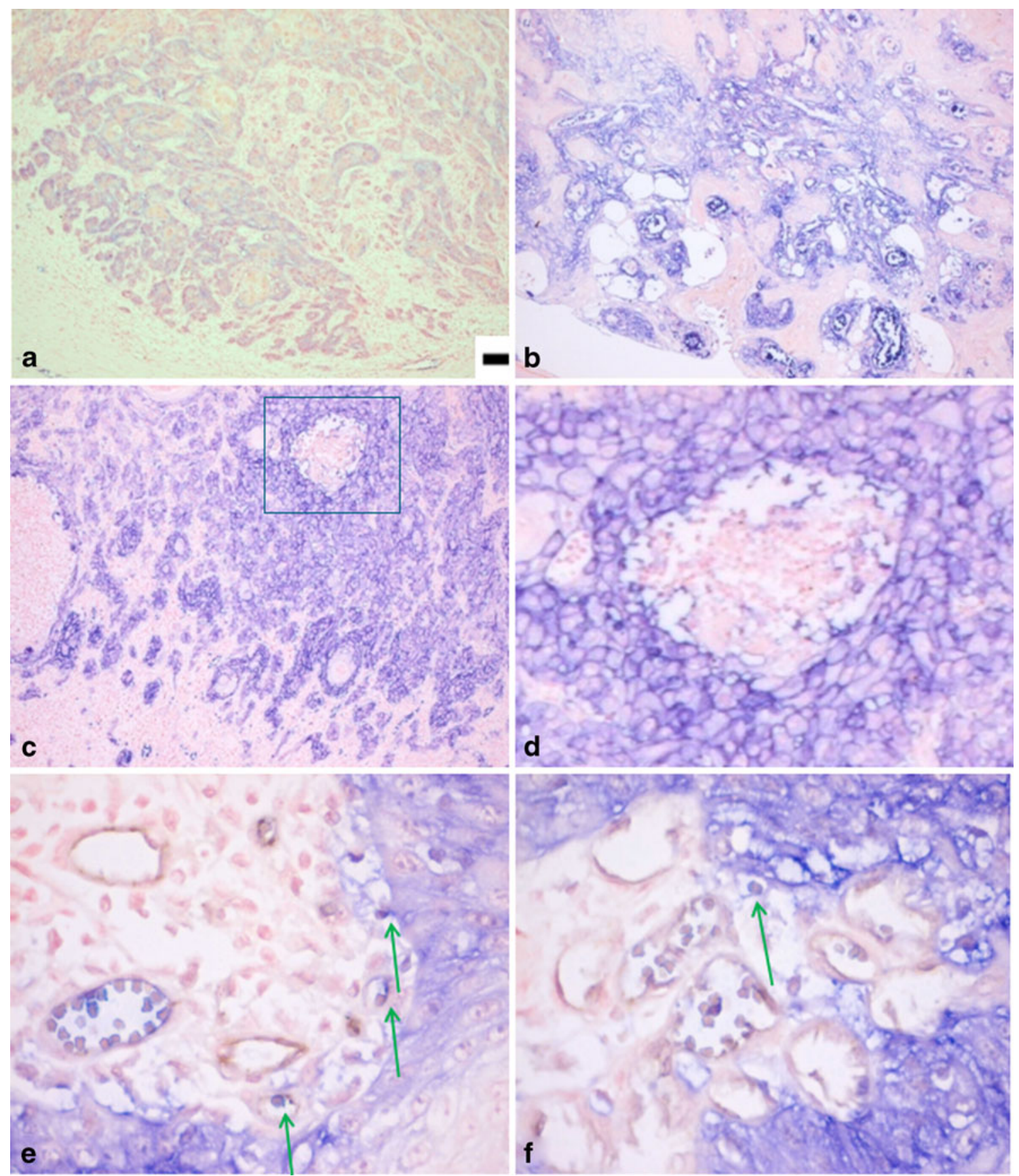

Fig. 1 GLUT-1 staining in vulvar squamous cell carcinomas. Representative image of a negative staining (a), 40\% (b), and 90\% positivity (c). High-power magnification (d) of indicated area in (c) demonstrates clear membranous localization. Scale bars (a)-(c): $50 \mu \mathrm{m},(\mathbf{d}): 10 \mu \mathrm{m}$. Membranous GLUT-1 staining of tumor cells

(blue) adjacent to erythrocyte-containing blood vessels (red) can be observed (e and f). Erythrocytes are GLUT-1 positive and serve as an internal control for positive staining. Microvessels, where diffusion can occur, are indicated (arrows)

GLUT-1, CA-IX, and preoperative Hb levels in relation to tumor characteristics

Tumor characteristics in relation to expression of GLUT-1 and CA-IX are summarized in Table 1. Less-differentiated tumors demonstrated lower GLUT-1 expression levels. There were no differences between tumor characteristics with respect to GLUT-1 and CA-IX expression at the invasive front of the tumors (data not shown).

Anemic patients $(<11.2 \mathrm{~g} / \mathrm{dL})$ presented with tumors with a larger diameter than patients with $\mathrm{Hb}$ levels within the normal range (Table 2). Subsequently, the correlation between GLUT-1 or CA-IX expression and $\mathrm{Hb}$ levels was determined. GLUT-1 and CA-IX were inversely correlated 



Fig. 2 CA-IX staining in vulvar squamous cell carcinomas. Representative image of a negative staining (a), 10\% (b), 60\% (c), and 90\% positivity (d). High-power magnification of indicated area in (d)

( $p=0.003,95 \% \mathrm{CI}-0.479$ to -0.104$)$. No correlation was found between preoperative $\mathrm{Hb}$ levels and CA-IX or GLUT-1 expression.

GLUT-1, CA-IX, and preoperative Hb levels in relation to presence of inguinofemoral metastases

For GLUT-1 and CA-IX expression patterns, no differences were found for presence of inguinofemoral or distant metastases (Fig. 3a, b). An imbalance between oxygen supply and consumption is more likely to occur in large tumors (large diameter or great depth of invasion). Therefore, we performed a subanalysis for large tumors (diameter $\geq 38 \mathrm{~mm}$ or depth of invasion $\geq 8 \mathrm{~mm}$, which are both the mean values). Expression of GLUT-1 and CA-IX did not differ between tumors smaller or greater than a diameter of $38 \mathrm{~mm}$ or between tumors with a depth of invasion smaller or greater than $8 \mathrm{~mm}$. In addition, GLUT-1 and CA-IX expression were not associated with presence of inguinofemoral or distant metastases in this subgroup of large tumors (data not shown).

For preoperative $\mathrm{Hb}$ levels, significant differences were found for presence of inguinofemoral metastases or distant demonstrates clear membranous localization. Scale bars (a)-(c): $50 \mu \mathrm{m},(\mathbf{d}): 10 \mu \mathrm{m}$

(log rank $=0.001)$ when stratified as in Table 2, and log rank $<0.001$ (Fig. 3c) when stratified between anemic patients $(\mathrm{Hb}<11.2 \mathrm{~g} / \mathrm{dL})$ and patients with normal $\mathrm{Hb}$ levels $(>11.2 \mathrm{~g} / \mathrm{dL})$.

GLUT-1, CA-IX, and preoperative Hb levels and disease-specific survival

Univariate Cox regression analysis showed that both GLUT-1 (HR 0.876, 95\% CI 0.583-1.115) and CA-IX (HR 1.086, 95\% CI 0.794-1.477) were not predictive for DSS, whereas preoperative $\mathrm{Hb}$ value was a prognostic factor $(p<0.001$, $\mathrm{HR}=0.146,95 \% \mathrm{CI} 0.059-0.358$ ). In addition, the number of positive inguionofemoral lymph nodes and development of groin or distant recurrence correlated with a poor DSS ( $p<$ $0.001)$. When these variables were included in a multivariate Cox proportional hazards analysis, preoperative $\mathrm{Hb}$ level turned out to be an independent prognostic factor for poor DSS $(p=0.021)$. These findings were confirmed in a linear regression analysis, demonstrating that groin, pelvic, or distant recurrences are independent of preoperative $\mathrm{Hb}$ level $(p=0.262)$. The Kaplan-Meier survival curves with corresponding log-rank values for GLUT-1, CA-IX, and $\mathrm{Hb}$ 


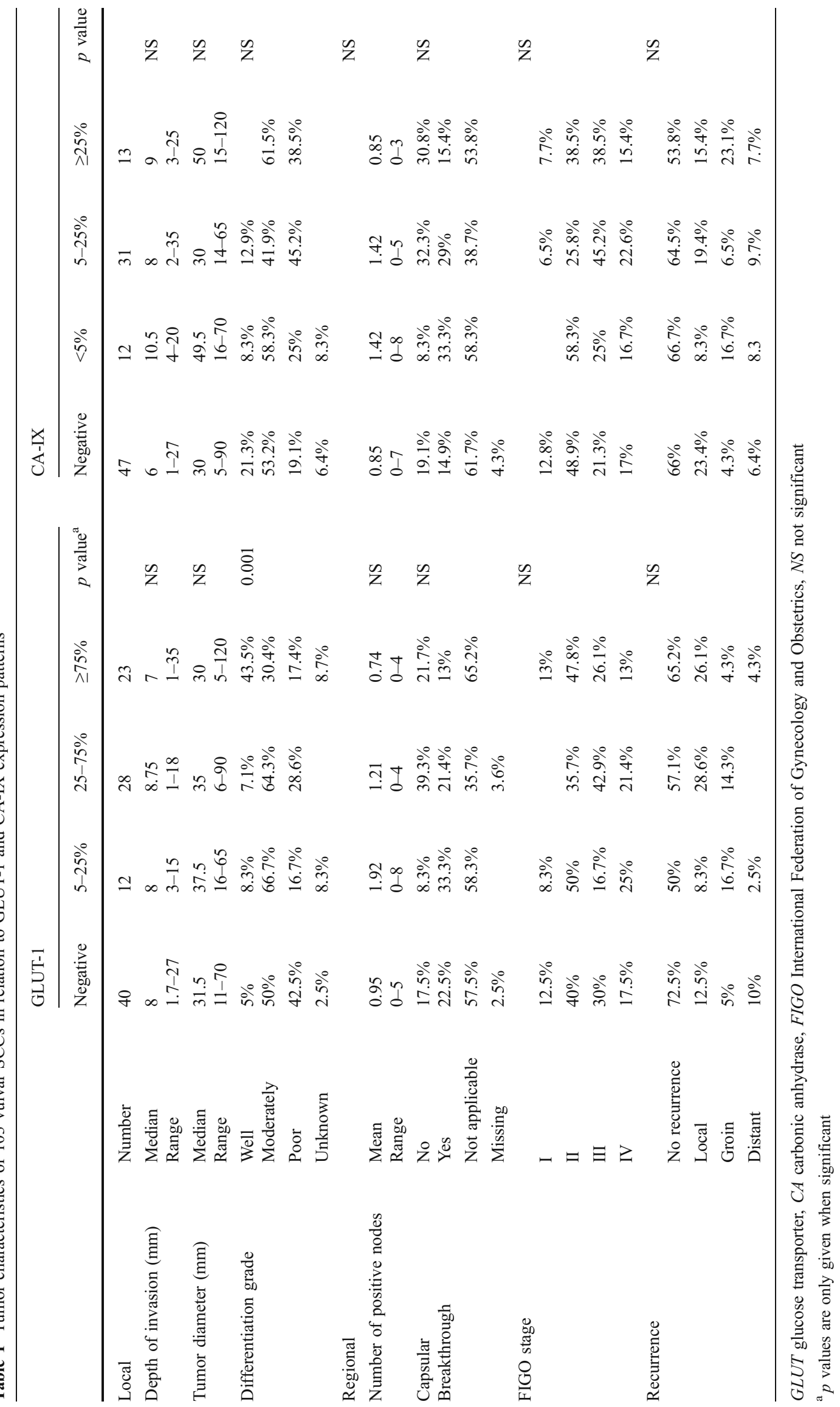


Table 2 Tumor characteristics of 77 vulvar SCCs in relation to preoperative Hb levels

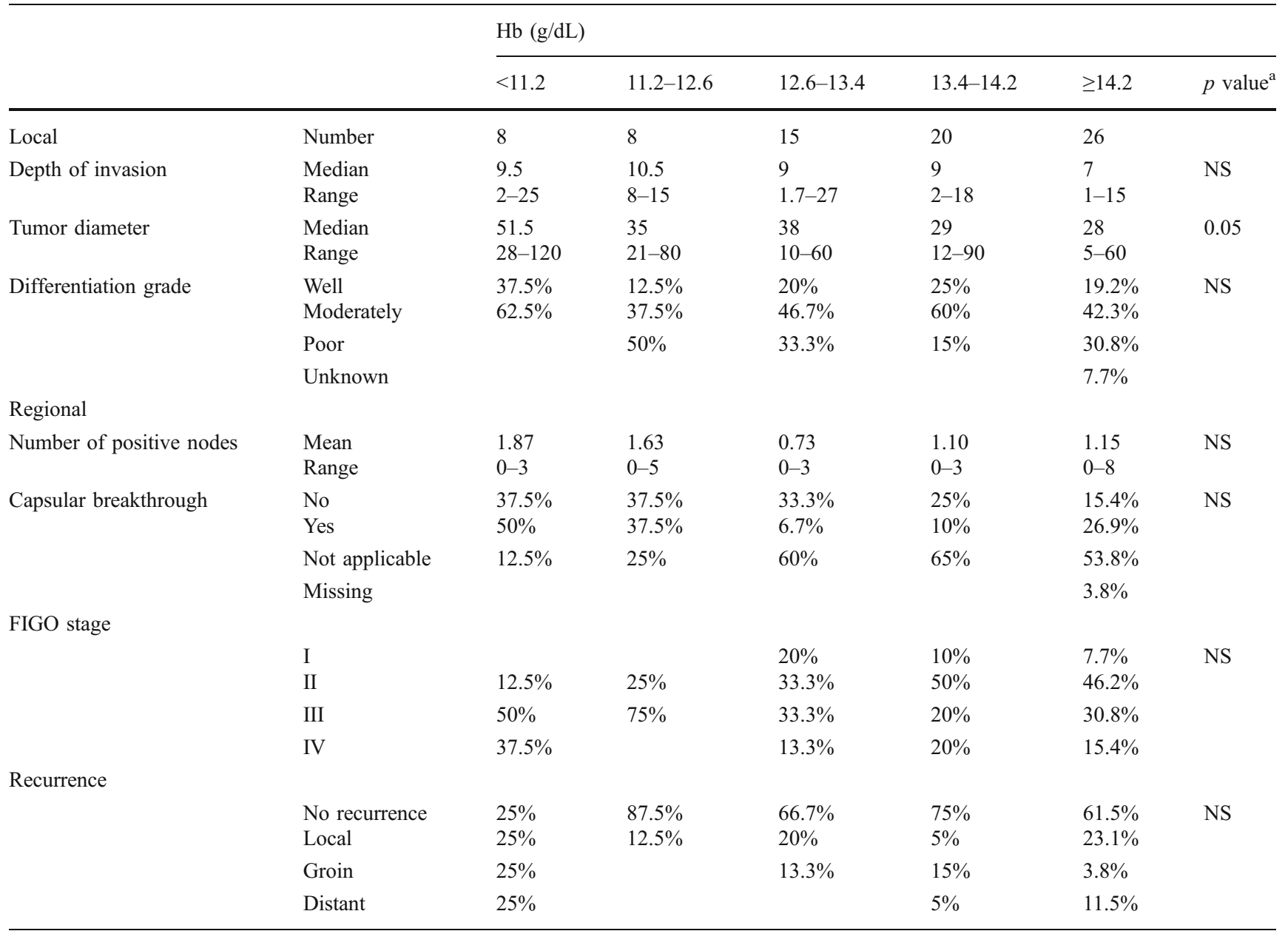

$H b$ hemoglobin, FIGO International Federation of Gynecology and Obstetrics, NS not significant

${ }^{a} p$ values are only given when significant; anemic patients were compared with non-anemic patients

level are shown in Fig. 4a-c. There was no difference in DSS between tumors with and without GLUT-1 and CA-IX expression at the invasive front (data not shown). This was also found in a subanalysis for patients treated with adjuvant radiotherapy (31 patients); preoperative $\mathrm{Hb}$ levels were predictive for DSS $(p=0.041)$, but not GLUT 1 and CAIX expression in the tumor ( $\log$ rank $p=0.507$ and 0.568 , respectively).

\section{Comorbidity}

The lack of a correlation between $\mathrm{Hb}$ levels and expression of hypoxia-associated markers provided no evidence for a link between anemia and tissue hypoxia. The correlation between $\mathrm{Hb}$ levels and poor DSS has led us to analyze whether $\mathrm{Hb}$ levels reflect the physical condition of the patients. Twenty-eight patients had no comorbidic conditions according to Charlson et al. [38], whereas one to six comorbidic conditions were observed in 75 patients (Table 3). The number of comorbidic conditions and $\mathrm{Hb}$ value were inversely associated in a linear regression model $(p=0.020,95 \%$ CI -0.295 to -0.026$)$.

\section{Discussion}

Our study of 103 patients with vulvar SCC did not reveal a prognostic value for the expression of the tissue hypoxiaassociated markers GLUT-1 and CA-IX. Although anemia has been linked to tissue hypoxia [14], we did not find such a correlation. However, low $\mathrm{Hb}$ values were associated with multiple comorbidic factors and turned out to be an independent prognostic factor for a poor DSS. Literature about the relation between the expression of hypoxiaassociated markers and lymph node metastasis or survival for vulvar SCCs patients is limited. A small-scale study 


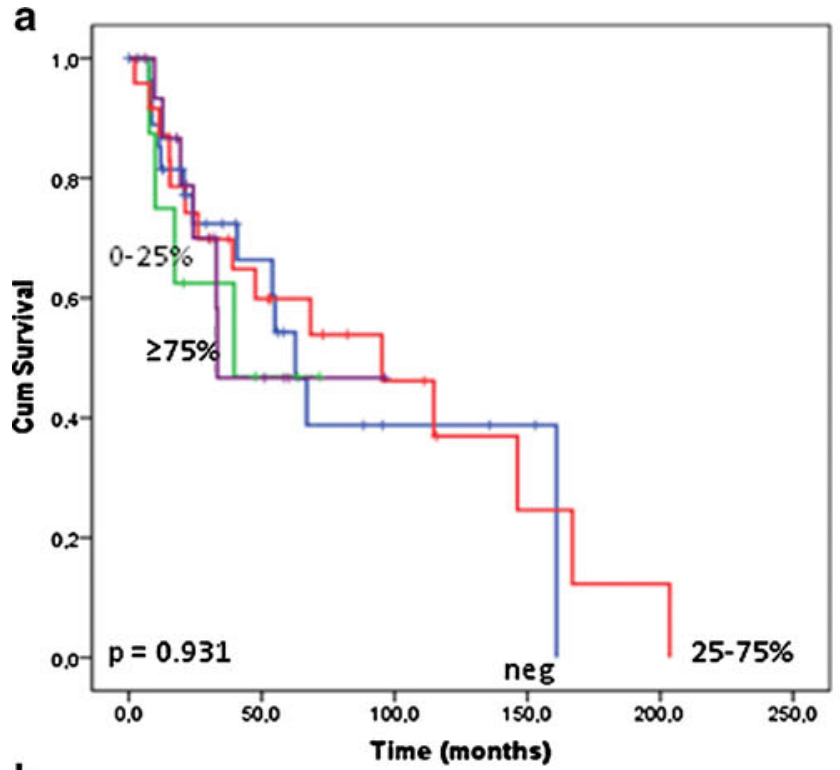

b
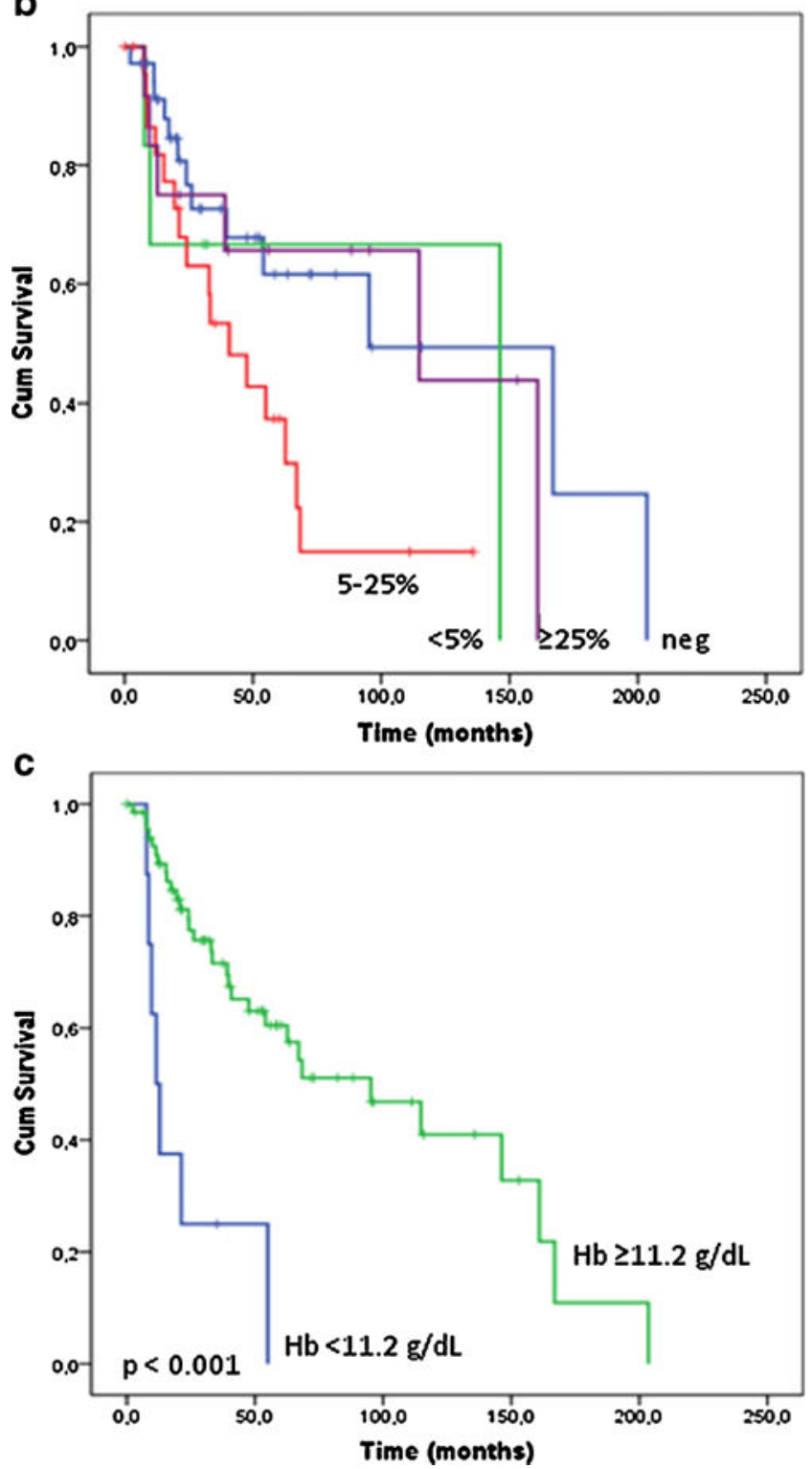

Fig. 3 Low preoperative Hb level correlates with increased risk of inguinofemoral or distant metastases. Presence of inguinofemoral or distant metastases curves for patients subdivided into different GLUT1 expression patterns (a), CA-IX patterns (b), and preoperative $\mathrm{Hb}$ levels (c). Low preoperative $\mathrm{Hb}$ levels significantly correlate with increased risk of inguinofemoral or distant metastases

comprising 20 patients likewise failed to demonstrate a significant correlation between various measures of tumor oxygenation and nodal metastasis [37].

Patients' anemia may contribute to the establishment of intratumoral hypoxic conditions by reducing oxygen delivery to tumor tissues. In our study, no correlation between $\mathrm{Hb}$ levels and hypoxia-associated markers could be demonstrated. Similarly, no association between $\mathrm{Hb}$ levels in patients before surgery and expression of hypoxia-associated markers was observed in 45 head and neck cancer and 79 colorectal adenocarcinomas [39, 40]. It is generally known that anemia has a negative impact on survival for cancer and non-cancer patients [41, 42]. However, previous research has focused on its correlation with hypoxia, but not as a representative for physical condition. We demonstrated an increased number of comorbidic conditions in vulvar SCC patients with low $\mathrm{Hb}$ values $(<11.2 \mathrm{~g} / \mathrm{dL})$, which in turn correlates with very poor prognosis (Fig. 4c). This is consistent with the study of $\mathrm{Wu}$ et al. who also found that the cohort of patients with anemia had the highest prevalences of several diseases [42], with even minimal deviations from an optimal range being associated with an increased risk of 30-day postoperative mortality [42]. A deterioration of prognosis with $\mathrm{Hb}$ levels below $11.2 \mathrm{~g} / \mathrm{dL}$ was also observed for cervical cancer patients treated with radiotherapy [43]. The differential diagnosis of anemia is extensive with chronic illness as a frequent cause. Cardiac and vascular conditions were most often present in our study population and may both be causes of the observed anemia in those patients. Because bleeding from a vulvar tumor rarely occurs, this factor is not likely to explain the low $\mathrm{Hb}$ levels in patients with large tumors. Therefore, it is more likely that low preoperative $\mathrm{Hb}$ levels represent an impaired physical condition than tumor hypoxia.

In our study, increasing percentages of GLUT-1 and CAIX expression were not associated with differences in primary tumor characteristics (Table 1), presence of inguinofemoral or distant metastases (Fig. 3), and DSS (Fig. 4). This may indicate that tumor hypoxia is not relevant for vulvar cancer or that the markers are of insufficient specificity. Tumor hypoxia can be induced by an inadequate function of the tumor vasculature independent of Hb levels. Furthermore, CA-IX and GLUT-1 do not necessarily behave in a coherent fashion [44] as we also observed in this study. The role of GLUT-1 as an endogenous marker for tumor hypoxia was found to be questionable [45]. Expression of GLUT-1 is induced by a 

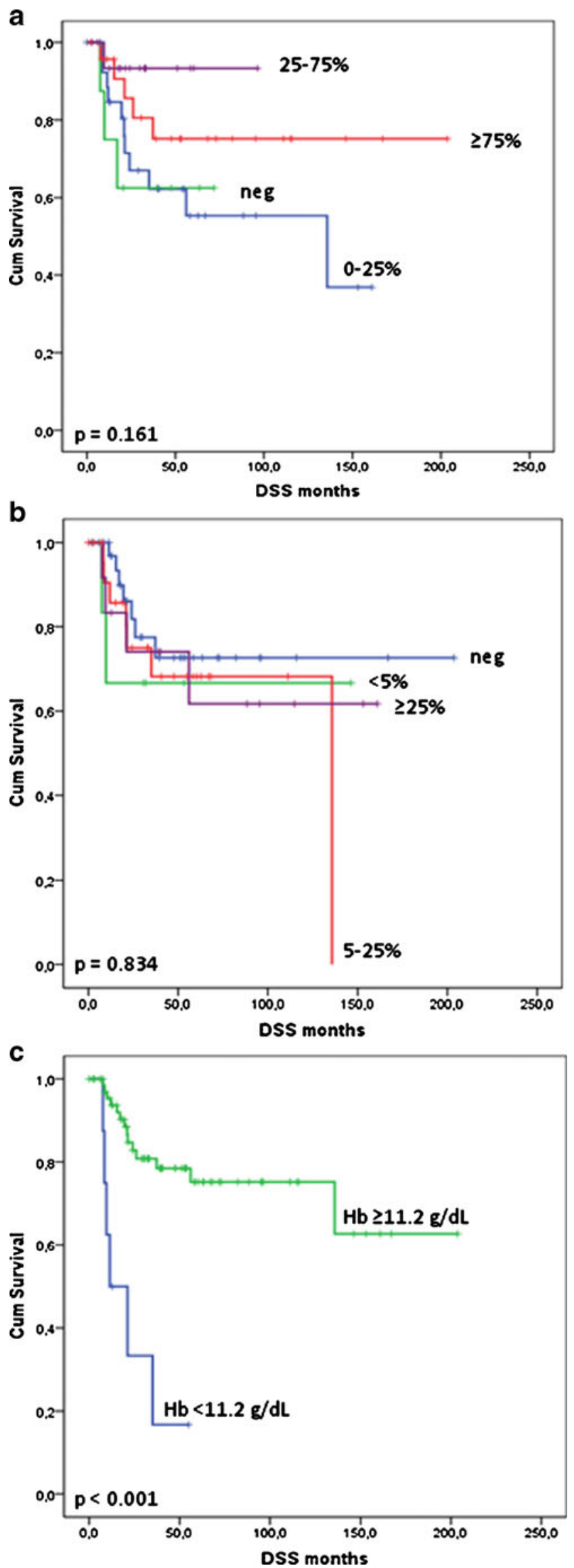

Fig. 4 Anemic vulvar SCC patients have a poor disease-free survival. Disease-free survival curves for patients subdivided into different GLUT-1 expression patterns (a), CA-IX patterns (b), and preoperative $\mathrm{Hb}$ levels (c). Low preoperative $\mathrm{Hb}$ levels significantly correlate with reduced DSS

variety of stimuli besides hypoxia, including glucose deprivation, oncogenic transformation, inhibition of oxidative phosphorylation, and osmotic stress [45]. Although GLUT expression has been demonstrated at a distance from vessels and adjacent to necrosis, the relationship with hypoxia has not been investigated [26]. Because we detected GLUT-1 expression directly adjacent to erythrocyte-containing blood vessel, we conclude that GLUT-1 is not a genuine hypoxia marker in vulvar SCC.

In prostate cancer, GLUT-1 mRNA expression correlates with loss of differentiation [46]. In contrast, we detected high GLUT-1 protein expression (i.e., $>75 \%$ ) more frequently in well-differentiated SCC than in moderate and poorly differentiated tumors (Table $1, p=0.001$ ). The relevance for vulvar SCC biology is currently unknown. However, this correlation is not observed in all other cases in which GLUT-1 is expressed by less than $75 \%$ of the tumor cells. It has to be noted, however, that our semiquantitative scoring of staining profiles is not as accurate as quantitative PCR. Small changes mRNA expression may have a prominent effect on tumor cell biology, but this altered gene expression may not be detected at the protein level in a semi-quantitative manner. CA-IX expression has been studied in a number of tumor types supporting the notion that CA-IX represents tumor aggressiveness. CA-IX is strongly induced by hypoxia in a broad range of tumor cells $[47,48]$ and considerable co-localization with pimonidazole staining was observed $[16,48]$. High expression of CA-IX is associated with poor survival in breast cancer, small cell lung cancer, head and neck and cervical tumors

Table 3 Overview of patients' comorbidity

\begin{tabular}{ll}
\hline Patients & 103 \\
No comorbidic conditions & 28 \\
Myocardial & 38 \\
Vascular & 54 \\
Pulmonary & 7 \\
Neurological & 6 \\
Endocrine & 26 \\
Renal & 1 \\
Liver & 1 \\
Gastrointestinal & 1 \\
Cancer & 11 \\
Miscellaneous & 7
\end{tabular}

The total number exceeds the total number of patients included (103) as some patients had multiple comorbidic conditions 
[30, 33-35], but an inverse correlation has been demonstrated for renal clear cell and rectal carcinomas [49-51], indicating that the prognostic or predictive value for CA-IX expression depends on tumor type. Our study does not indicate prognostic value of CA-IX expression for vulvar SCC patients.

In conclusion, we found that high GLUT-1 and CA-IX protein expression levels do not predict DSS for vulvar SCC patients. Furthermore, their expression did not correlate with preoperative $\mathrm{Hb}$ levels. However, preoperative $\mathrm{Hb}$ levels could predict DSS independently of the development of a groin or distant recurrence, and also correlated with the number of comorbidic conditions. It is most likely that preoperative $\mathrm{Hb}$ levels represent overall physical condition more than it predicts tumor hypoxia.

Acknowledgment We thank Dr. J. Hendriks (Department of Epidemiology and Biostatistics, Radboud University Nijmegen Medical Centre) for his expert help regarding the statistical methods used in this study.

\section{Disclosure/conflict of interest None}

Open Access This article is distributed under the terms of the Creative Commons Attribution Noncommercial License which permits any noncommercial use, distribution, and reproduction in any medium, provided the original author(s) and source are credited.

\section{References}

1. Lataifeh I, Nascimento MC, Nicklin JL, Perrin LC, Crandon AJ, Obermair A (2004) Patterns of recurrence and disease-free survival in advanced squamous cell carcinoma of the vulva. Gynecol Oncol 95(3):701-705

2. Preti M, Ronco G, Ghiringhello B, Micheletti L (2000) Recurrent squamous cell carcinoma of the vulva: clinicopathologic determinants identifying low risk patients. Cancer 88(8):1869-1876

3. Raspagliesi F, Hanozet F, Ditto A, Solima E, Zanaboni F, Vecchione $F$ et al (2006) Clinical and pathological prognostic factors in squamous cell carcinoma of the vulva. Gynecol Oncol 102(2):333-337

4. Oonk MH, Hollema H, de Hullu JA, van der Zee AG (2006) Prediction of lymph node metastases in vulvar cancer: a review. Int J Gynecol Cancer 16(3):963-971

5. van der Zee AG, Oonk MH, de Hullu JA, Ansink AC, Vergote I, Verheijen RH et al (2008) Sentinel node dissection is safe in the treatment of early-stage vulvar cancer. J Clin Oncol 26(6):884-889

6. Fons G, Burger MP, ten Kate FJ, van der Velden (2007) Identification of potential prognostic markers for vulvar cancer using immunohistochemical staining of tissue microarrays. Int $\mathbf{J}$ Gynecol Pathol 26(2):188-193

7. Growdon WB, Boisvert SL, Akhavanfard S, Oliva E, as-Santagata DC, Kojiro S et al (2008) Decreased survival in EGFR gene amplified vulvar carcinoma. Gynecol Oncol 111(2):289-297

8. Johnson GA, Mannel R, Khalifa M, Walker JL, Wren M, Min KW et al (1997) Epidermal growth factor receptor in vulvar malignancies and its relationship to metastasis and patient survival. Gynecol Oncol 65(3):425-429
9. Nayha VV, Stenback FG (2007) Increased angiogenesis is associated with poor prognosis of squamous cell carcinoma of the vulva. Acta Obstet Gynecol Scand 86(11):1392-1397

10. Obermair A, Kohlberger P, Bancher-Todesca D, Tempfer C, Sliutz $\mathrm{G}$, Leodolter S et al (1996) Influence of microvessel density and vascular permeability factor/vascular endothelial growth factor expression on prognosis in vulvar cancer. Gynecol Oncol 63 (2):204-209

11. Oonk MH, de Bock GH, van der Veen T, Hoor KA, de Hullu JA, Hollema $\mathrm{H}$ et al (2007) EGFR expression is associated with groin node metastases in vulvar cancer, but does not improve their prediction. Gynecol Oncol 104(1):109-113

12. Varlotto J, Stevenson MA (2005) Anemia, tumour hypoxemia, and the cancer patient. Int J Radiat Oncol Biol Phys 63(1):25-36

13. Qutub AA, Popel AS (2007) Three autocrine feedback loops determine HIF1 alpha expression in chronic hypoxia. Biochim Biophys Acta 1773(10):1511-1525

14. Hoogsteen IJ, Marres HA, Bussink J, van der Kogel AJ, Kaanders JH (2007) Tumour microenvironment in head and neck squamous cell carcinomas: predictive value and clinical relevance of hypoxic markers. A review. Head Neck 29(6):591-604

15. Hockel M, Vorndran B, Schlenger K, Baussmann E, Knapstein PG (1993) Tumour oxygenation: a new predictive parameter in locally advanced cancer of the uterine cervix. Gynecol Oncol 51 (2):141-149

16. Kaanders JH, Wijffels KI, Marres HA, Ljungkvist AS, Pop LA, van den Hoogen FJ et al (2002) Pimonidazole binding and tumour vascularity predict for treatment outcome in head and neck cancer. Cancer Res 62(23):7066-7074

17. Sundfor K, Lyng H, Rofstad EK (1998) Tumour hypoxia and vascular density as predictors of metastasis in squamous cell carcinoma of the uterine cervix. Br J Cancer 78(6):822-827

18. Gnarra JR, Zhou S, Merrill MJ, Wagner JR, Krumm A, Papavassiliou E et al (1996) Post-transcriptional regulation of vascular endothelial growth factor mRNA by the product of the VHL tumour suppressor gene. Proc Natl Acad Sci USA 93 (20):10589-10594

19. Hanahan D, Folkman J (1996) Patterns and emerging mechanisms of the angiogenic switch during tumourigenesis. Cell 86(3):353-364

20. Ivanov SV, Kuzmin I, Wei MH, Pack S, Geil L, Johnson BE et al (1998) Down-regulation of transmembrane carbonic anhydrases in renal cell carcinoma cell lines by wild-type von Hippel-Lindau transgenes. Proc Natl Acad Sci USA 95(21):12596-12601

21. Maxwell PH, Wiesener MS, Chang GW, Clifford SC, Vaux EC, Cockman ME et al (1999) The tumour suppressor protein VHL targets hypoxia-inducible factors for oxygen-dependent proteolysis. Nature 399(6733):271-275

22. Macheda ML, Rogers S, Best JD (2005) Molecular and cellular regulation of glucose transporter (GLUT) proteins in cancer. J Cell Physiol 202(3):654-662

23. Sly WS, Hu PY (1995) Human carbonic anhydrases and carbonic anhydrase deficiencies. Annu Rev Biochem 64:375-401

24. Ivanov S, Liao SY, Ivanova A, Danilkovitch-Miagkova A, Tarasova N, Weirich G et al (2001) Expression of hypoxiainducible cell-surface transmembrane carbonic anhydrases in human cancer. Am J Pathol 158(3):905-919

25. Vaupel P, Mayer A (2005) Hypoxia and anemia: effects on tumour biology and treatment resistance. Transfus Clin Biol 12(1):5-10

26. Hoogsteen IJ, Marres HA, van der Kogel AJ, Kaanders JH (2007) The hypoxic tumour microenvironment, patient selection and hypoxia-modifying treatments. Clin Oncol (R Coll Radiol) 19 (6):385-396

27. Kelleher DK, Mattheinsen U, Thews O, Vaupel P (1996) Blood flow, oxygenation, and bioenergetic status of tumours after erythropoietin treatment in normal and anemic rats. Cancer Res 56(20):4728-4734 
28. Airley R, Loncaster J, Davidson S, Bromley M, Roberts S, Patterson A et al (2001) Glucose transporter glut-1 expression correlates with tumour hypoxia and predicts metastasis-free survival in advanced carcinoma of the cervix. Clin Cancer Res 7 (4):928-934

29. Cantuaria G, Fagotti A, Ferrandina G, Magalhaes A, Nadji M, Angioli R et al (2001) GLUT-1 expression in ovarian carcinoma: association with survival and response to chemotherapy. Cancer 92(5):1144-1150

30. Chia SK, Wykoff CC, Watson PH, Han C, Leek RD, Pastorek J et al (2001) Prognostic significance of a novel hypoxia-regulated marker, carbonic anhydrase IX, in invasive breast carcinoma. J Clin Oncol 19(16):3660-3668

31. Fortin A, Wang CS, Vigneault E (2008) Effect of pretreatment anemia on treatment outcome of concurrent radiochemotherapy in patients with head and neck cancer. Int J Radiat Oncol Biol Phys 72(1):255-260

32. Furudoi A, Tanaka S, Haruma K, Yoshihara M, Sumii K, Kajiyama $G$ et al (2001) Clinical significance of human erythrocyte glucose transporter 1 expression at the deepest invasive site of advanced colorectal carcinoma. Oncology 60 (2):162-169

33. Giatromanolaki A, Koukourakis MI, Sivridis E, Turley H, Talks $\mathrm{K}$, Pezzella F et al (2001) Relation of hypoxia inducible factor 1 alpha and 2 alpha in operable non-small cell lung cancer to angiogenic/molecular profile of tumours and survival. Br J Cancer 85(6):881-890

34. Koukourakis MI, Giatromanolaki A, Sivridis E, Simopoulos K, Pastorek J, Wykoff CC et al (2001) Hypoxia-regulated carbonic anhydrase-9 (CA9) relates to poor vascularization and resistance of squamous cell head and neck cancer to chemoradiotherapy. Clin Cancer Res 7(11):3399-3403

35. Loncaster JA, Harris AL, Davidson SE, Logue JP, Hunter RD, Wycoff CC et al (2001) Carbonic anhydrase (CA IX) expression, a potential new intrinsic marker of hypoxia: correlations with tumour oxygen measurements and prognosis in locally advanced carcinoma of the cervix. Cancer Res 61(17):6394-6399

36. Winter WE III, Maxwell GL, Tian C, Sobel E, Rose GS, Thomas $G$ et al (2004) Association of hemoglobin level with survival in cervical carcinoma patients treated with concurrent cisplatin and radiotherapy: a Gynecologic Oncology Group Study. Gynecol Oncol 94(2):495-501

37. Stone JE, Parker R, Gilks CB, Stanbridge EJ, Liao SY, QuinoParsons C (2005) Intratumoural oxygenation of invasive squamous cell carcinoma of the vulva is not correlated with regional lymph node metastasis. Eur J Gynaecol Oncol 26(1):31-35

38. Charlson ME, Pompei P, Ales KL, MacKenzie CR (1987) A new method of classifying prognostic comorbidity in longitudinal studies: development and validation. J Chronic Dis 40 (5):373-383
39. Koukourakis MI, Giatromanolaki A, Sivridis E, Pastorek J, Karapantzos I, Gatter KC et al (2004) Hypoxia-activated tumour pathways of angiogenesis and $\mathrm{pH}$ regulation independent of anemia in head-and-neck cancer. Int J Radiat Oncol Biol Phys 59(1):67-71

40. Koukourakis MI, Giatromanolaki A, Polychronidis A, Simopoulos C, Gatter KC, Harris AL et al (2006) Endogenous markers of hypoxia/anaerobic metabolism and anemia in primary colorectal cancer. Cancer Sci 97(7):582-588

41. Caro JJ, Salas M, Ward A, Goss G (2001) Anemia as an independent prognostic factor for survival in patients with cancer: a systemic, quantitative review. Cancer 91(12):2214-2221

42. Wu WC, Schifftner TL, Henderson WG, Eaton CB, Poses RM, Uttley G et al (2007) Preoperative hematocrit levels and postoperative outcomes in older patients undergoing noncardiac surgery. JAMA 297(22):2481-2488

43. Dunst J, Kuhnt T, Strauss HG, Krause U, Pelz T, Koelbl H et al (2003) Anemia in cervical cancers: impact on survival, patterns of relapse, and association with hypoxia and angiogenesis. Int $\mathrm{J}$ Radiat Oncol Biol Phys 56(3):778-787

44. Jonathan RA, Wijffels KI, Peeters W, de Wilde PC, Marres HA, Merkx MA et al (2006) The prognostic value of endogenous hypoxia-related markers for head and neck squamous cell carcinomas treated with ARCON. Radiother Oncol 79(3):288-297

45. Mayer A, Hockel M, Wree A, Vaupel P (2005) Microregional expression of glucose transporter-1 and oxygenation status: lack of correlation in locally advanced cervical cancers. Clin Cancer Res 11(7):2768-2773

46. Stewart GD, Gray K, Pennington CJ, Edwards DR, Riddick AC, Ross JA et al (2008) Analysis of hypoxia-associated gene expression in prostate cancer: lysyl oxidase and glucose transporter-1 expression correlate with Gleason score. Oncol Rep 20(6):1561-1567

47. Lal A, Peters H, St CB, Haroon ZA, Dewhirst MW, Strausberg RL et al (2001) Transcriptional response to hypoxia in human tumours. J Natl Cancer Inst 93(17):1337-1343

48. Wykoff CC, Beasley NJ, Watson PH, Turner KJ, Pastorek J, Sibtain A et al (2000) Hypoxia-inducible expression of tumourassociated carbonic anhydrases. Cancer Res 60(24):7075-7083

49. Bui MH, Seligson D, Han KR, Pantuck AJ, Dorey FJ, Huang Y et al (2003) Carbonic anhydrase IX is an independent predictor of survival in advanced renal clear cell carcinoma: implications for prognosis and therapy. Clin Cancer Res 9(2):802-811

50. Murakami Y, Kanda K, Tsuji M, Kanayama H, Kagawa S (1999) $\mathrm{MN} / \mathrm{CA} 9$ gene expression as a potential biomarker in renal cell carcinoma. BJU Int 83(7):743-747

51. Rasheed S, Harris AL, Tekkis PP, Turley H, Silver A, McDonald PJ et al (2009) Assessment of microvessel density and carbonic anhydrase-9 (CA-9) expression in rectal cancer. Pathol Res Pract 205(1):1-9 